\title{
Statically constrained nonlinear models with application to IC buffers
}

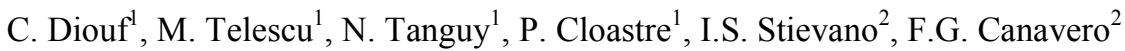 \\ ${ }^{1}$ Université Européenne de Bretagne, France. Université de Brest ; CNRS, UMR 3192 Lab-STICC, ISSTB \\ ${ }^{2}$ Politecnico di Torino, Italy \\ Reference contact: mihai.telescu@univ-brest.fr
}

\begin{abstract}
Volterra series are well known approaches to modeling non-linear systems. In recent works a properly weighted combination of Volterra models have been used to successfully mimic the behavior of the output port of an integrated circuit buffer. The current paper focuses on a novel mechanism for controlling the static characteristic of such models. A commercial driver example is used to illustrate the efficiency of the technique to guarantee accurate static levels during time-domain simulations.
\end{abstract}

\section{Introduction}

The modeling of digital Integrated Circuits (IC) input and output buffers is a vital stage in the assessment of signal integrity and electromagnetic compatibility in high-end digital systems. Buffers both drivers and receivers act as non-linear terminals of the interconnect line networks and their transient behavior may have unwanted effects on the proper functioning of the system. In recent years, researchers have concentrated their attention on the development of behavioral models that seek to mimic the device's input-output behavior of ten proves more efficient from a computational point of view than attempting to model its internal physics.

Different approaches exist ranging from neural networks [1] to more common two-piece models [2-3] exploiting the two-state nature of the device. In a previous paper [4] some of the authors showed that two properly weighted weakly nonlinear Volterra models could successfully be used to mimic the output behavior of drivers. The strength of this approach resides in a greater degree of generality allowing for greater adaptability.

In this paper the authors focus on the static characteristic of Volterra models and the way in which they may be forced to fit a certain form. This is particularly interesting in the field of buffer modeling where the static behavior of the original system needs to be accurately reproduced.

\section{Volterra and Volterra-Laguerre models}

Volterra series are a well-known input-output representation of nonlinear systems and have found in the last half-century a variety of applications in different fields of science. They have proved to be a valuable and reliable tool in nonlinear system identification and allow a straightforward generalization to multivariable systems. The general discrete time expression of a Volterra series is given by

$$
\begin{aligned}
y(k)= & \sum_{k_{1}=0}^{\infty} h_{1}\left(k_{1}\right) x\left(k-k_{1}\right) \\
& +\sum_{k_{1}=0}^{\infty} \sum_{k_{2}=0}^{\infty} h_{2}\left(k_{1}, k_{2}\right) x\left(k-k_{1}\right) x\left(k-k_{2}\right) \\
& +\ldots \\
= & \sum_{m=1}^{\infty} \sum_{k_{1}=0}^{\infty} \ldots \sum_{k_{m}=0}^{\infty} h_{m}\left(k_{1}, k_{2}, \ldots, k_{m}\right) \prod_{l=1}^{m} x\left(k-k_{l}\right)
\end{aligned}
$$

with $k$ being the discrete time, $y$ the output variable, $x$ the input and $h_{m}$ being referred to as the $m$-th order kernel. One may intuitively see Volterra series as a generalization of a linear systems impulse response function and it is quite obvious that for $m=1$ expression (1) is reduced to the classical impulse response function of a linear system.

In practice direct identification of the kernels, for an unknown nonlinear system, may prove cumbersome. A common approach is to use a projection on an orthogonal basis. The discrete Laguerre basis has been chosen to illustrate the approach presented in this paper, although the mathematical derivations may be extended to other representations.

The discrete Laguerre functions $\phi_{i}(k)$ may be defined by their $z$ transform as follows

$$
\Phi_{i}(z)=\sqrt{1-a^{2}} \frac{z}{z-a}\left(\frac{1-a z}{z-a}\right)^{i}, \quad i=0,1, \ldots
$$

Laguerre functions form a complete set of orthonormal functions in $\ell^{2}\left(\Re_{+}\right)$, then each Volterra kernel can be expressed with a multidimensional Laguerre series as

$$
\left\{\begin{array}{l}
h_{1}\left(k_{1}\right)=\sum_{i_{1}=0}^{\infty} C_{i_{1}} \phi_{i_{1}}\left(k_{1}\right) \\
h_{2}\left(k_{1}, k_{2}\right)=\sum_{i_{1}=0}^{\infty} \sum_{i_{2}=0}^{\infty} C_{i_{1}, i_{2}} \phi_{i_{1}}\left(k_{1}\right) \phi_{i_{2}}\left(k_{2}\right) \\
\ldots
\end{array}\right.
$$

Let $\bar{\phi}_{i}(k)$ denote the response of the $i$ th Laguerre filter with transfer function given by (2) to the input $x(k)$, i.e.

$$
\bar{\phi}_{i}(k)=\phi_{i}(k) * x(k)=\sum_{k_{l}=0}^{\infty} \phi_{i}\left(k_{l}\right) x\left(k-k_{l}\right) .
$$

It follows that equation (1) can be recast as 


$$
\begin{aligned}
y(k)= & \sum_{i_{1}=0}^{\infty} C_{i_{1}} \bar{\phi}_{i_{1}}(k) \\
& +\sum_{i_{1}=0}^{\infty} \sum_{i_{2}=0}^{\infty} C_{i_{1}, i_{2}} \bar{\phi}_{i_{1}}(k) \bar{\phi}_{i_{2}}(k) \\
& +\ldots \\
= & \sum_{m=1}^{\infty} \sum_{i_{1}=0}^{\infty} \ldots \sum_{i_{m}=0}^{\infty} C_{i_{1} i_{2}, \ldots, i_{m}} \prod_{l=1}^{m} \bar{\phi}_{i_{l}}(k)
\end{aligned}
$$

Thus, with a known well chosen input signal and the respective, known, output signal one may compute the expansion coefficients $C_{i_{1}}, C_{i_{1}, i_{2}}, \ldots, C_{i_{1}, i_{2} \ldots i_{m}}$. Subsequently the response to any input signal may be obtained.

In practice a finite number of kernels is considered $m<M$, and the expansion of each kernel is truncated at an order $N_{m}$. Due to the truncation error the choice of parameter $a$ in (2) is non trivial. Further more, different parameters can be used for the different kernels. These aspects are discussed in [5] and [6].

\section{The static characteristic of a Volterra-Laguerre model}

It may be shown that the static characteristic of a VolterraLaguerre model is a polynomial given by the $z$-transform of $y(k)$ for $z=\exp (j \omega)$ at $\omega=0$, i.e

$$
Y\left(e^{j \cdot 0}\right)=p_{1} A+p_{2} A^{2}+\ldots+p_{M} A^{M}
$$

where $A$ denotes the amplitude of the input and

$$
p_{m}=\left(\sqrt{\frac{1+a}{1-a}}\right)^{m} \sum_{i_{1}=0}^{N_{m}-1} \sum_{i_{2}=0}^{N_{m}-1} \ldots \sum_{i_{m}=0}^{N_{m}-1} C_{i_{1}, i_{2}, \ldots, i_{m}}, .
$$

for $m=1,2, \ldots M$.

Let us now suppose that we wish to optimally modify the coefficients of the expansion with the idea to force a new static characteristic given by

$$
Y^{\prime}\left(e^{j \cdot 0}\right)=\sigma_{1} A+\sigma_{2} A^{2}+\ldots+\sigma_{M} A^{M} .
$$

Correcting each coefficient of the polynomial is a classical optimization problem under linear constraints of the type $L_{m} \vec{C}_{m}-\sigma_{m}$ that can be solved using Lagrange multipliers. In [7] it has been shown that in the particular cases of orthogonal projections the solution is given by

$$
\vec{C}_{m}^{\prime}=\vec{C}_{m}-L_{m}^{T}\left(L_{m} L_{m}^{T}\right)^{-1}\left(L_{m} \vec{C}_{m}-\sigma_{m}\right), \quad m=1,2, \ldots M .
$$

where vectors $\vec{C}_{m}$ and $\vec{C}_{m}^{\prime}$ respectively stack all the uncorrected and corrected coefficients of the $\mathrm{m}^{\text {th }}$ order kernel

$$
\vec{C}_{m}=\left[C_{0, \ldots, 0,0}, C_{0, \ldots, 0,1}, \ldots, C_{N_{m}-1, \ldots, N_{m-1}-1, N_{m}-1}\right]^{T},
$$

and line vector $L_{m}$ contains the constraint

$$
L_{m}=\left(\sqrt{\frac{1+a}{1-a}}\right)^{m} \cdot[1,1, \ldots, 1] .
$$

After simplification the solution is given by the following correction of individual coefficients

$$
C_{i_{1}, i_{2}, \ldots, i_{m}}^{\prime}=C_{i_{1}, i_{2}, \ldots, i_{m}}-\left(\sqrt{\frac{1-a}{1+a}}\right)^{m} \frac{1}{N_{m}^{m}}\left(p_{m}-\sigma_{m}\right) .
$$

\section{Two-state Volterra models for IC buffers}

The method presented in [2] seeks to model the output port of a buffer (fig. 1) by expressing the output current $i(t)$ as a weighted sum of $i_{H}(t)$ and $i_{L}(t)$ respectively corresponding to the high-state and the low-state output currents. Each term non-linearly depends on the output voltage $v(t)$. The expression of the output current is then given by

$$
i(t)=w_{H}(t) i_{H}(v(t), t)+w_{L}(t) i_{L}(v(t), t) .
$$

This assumption is justified by the inclusion in the model equations of the inherent logical switching operation of this class of devices. This solution has been demonstrated to provide good results in terms of both model accuracy and model efficiency.

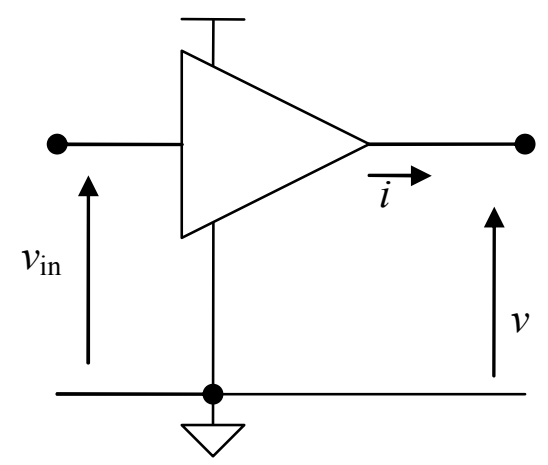

Fig. 1. Single ended output buffer.

As presented in [4], each sub-system, non-linearly binding $i_{H}(t)$ to $v(t)$ and

$i_{L}(t)$ to $v(t)$ may then be approximated by a VolterraLaguerre model.

At this stage the new technique described in the previous section may be used to guarantee a good static characteristic of the buffer model. Note that in practice buffer static characteristics are not necessarily polynomial. Furthermore, even if one accounts for worst case scenarios only a part of the characteristic will be explored in practice. Therefore the best algorithm to be used is

1) For each sub-model (high state and low state) identify the region of interest of the static characteristic.

2) Fit the characteristic with a polynomial according to (8).

3) Use (10) to correct the coefficients of the VolterraLaguerre expansions to fit the desired characteristic.

In the case of differential drivers (fig. 2), one has to account for the two output ports. Thus, eq. (11) needs to be generalized as follows

$$
i_{\lambda}(t)=w_{\lambda, H}(t) i_{\lambda, H}\left(v_{c}(t), v_{d}(t), t\right)+w_{\lambda, L}(t) i_{\lambda, L}\left(v_{c}(t), v_{d}(t), t\right)
$$

where $\lambda=1,2, \quad v_{c}=\left(v_{1}+v_{2}\right) / 2 \quad$ and $\quad v_{d}=\left(v_{1}-v_{2}\right)$ are respectively the common and differential mode voltages. 


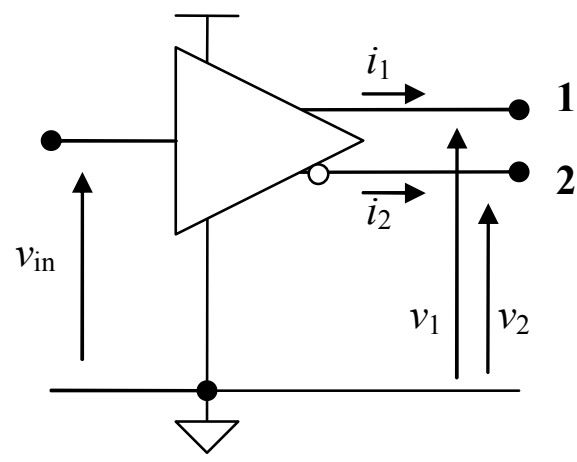

Fig.2. Differential driver.

The generalization of the Volterra-Laguerre expansion given by (5) is straightforward but leads to a lengthy expression which is why it was presented in the appendix. Obviously the static characteristic will in this case be bi-dimensional and its correction will require a bi-dimensional fit. This generalization does not pose any particular problems, it relates to basic spatial geometry. The last step of the algorithm remains essentially unchanged; the correction of individual coefficients is performed in the same way.

\section{Simulation results}

In most cases the identification technique used in [4] works well on most drivers and the "blindly" identified static characteristic is reproduced with sufficient accuracy. Nonetheless, as shown in the following example, in some cases, particularly when a very compact model is computed, the static characteristic of the model may diverge from that of the original system. In the event of such scenarios, the procedure described in this paper becomes a useful and elegant tool. The user is no longer forced to increase the model complexity (i.e. the truncation order of the kernels) but may simply resort to a statically constrained solution.

A commercial differential driver was used for the tests presented below. Its two output ports were modeled using a two-kernel Volterra-Laguerre model with a total of four Laguerre functions for the 1st order kernel and four for the 2nd order. Figures $3 \mathrm{a}$ ) and $3 \mathrm{~b}$ ) show the common mode voltage $v_{c}$ sweep of the on-state static characteristic of the driver for different values of the differential voltage $v_{d}$. Figure 4 shows the result of a dynamic simulation tracing the differential mode response of both the original system and the model for a "010" sequence. It should be noted that the correction of the static characteristic is effective well outside the working range of the device as shown in figure 3 and that it is achieved without visible damage to the dynamic performance as seen in figure 4 . Indeed, the overshoots and undershoots are accurately reproduced.

\section{Conclusion}

In the behavioral modeling of digital IC buffers one needs to show particular care in accurately reproducing the static characteristics of the devices. This short paper addresses the problem of computing buffer models using Volterra-Laguerre series while guaranteeing a correct static characteristic. The key feature is an a posteriori optimal correction technique which enables the user to analytically force a specific static characteristic on the model without damaging its dynamic qualities.
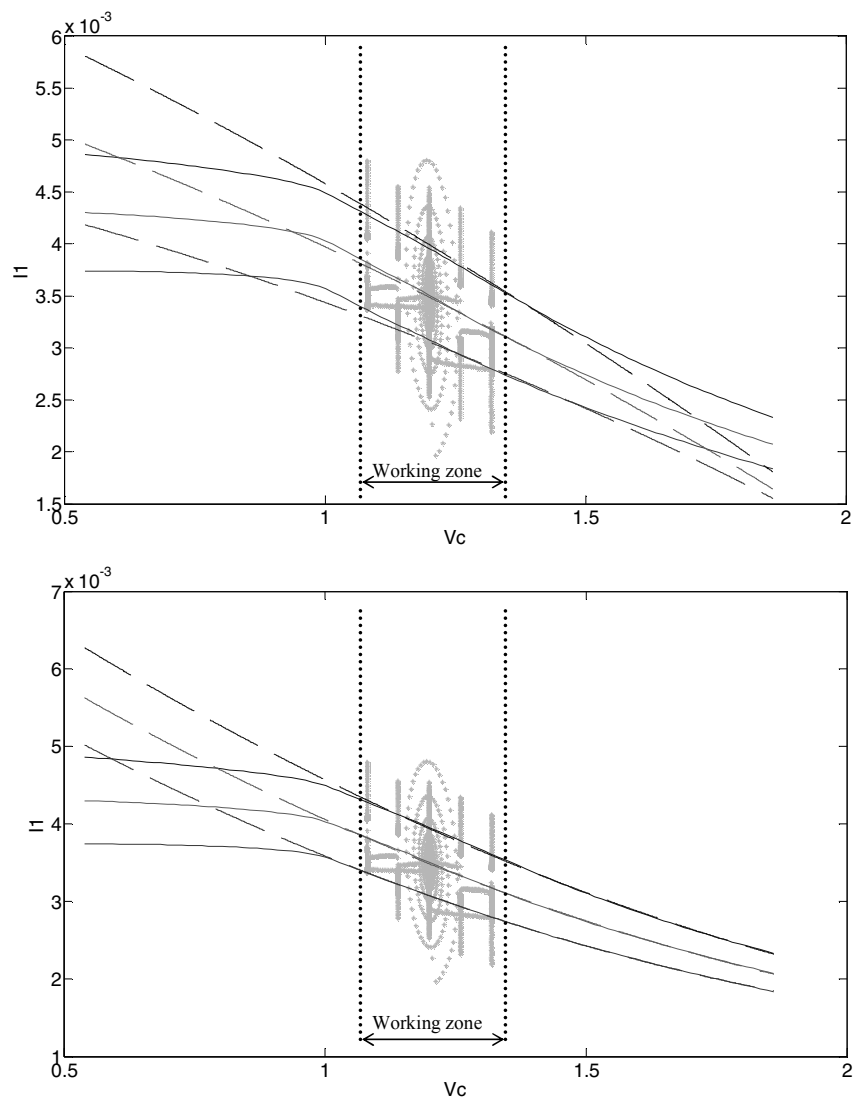

Fig. 3a and 3b. Solid lines: static characteristics of a commercial driver: output current $i_{1}$ versus common mode voltage $v_{c}$ for different values of the differential voltage $v_{d}$. Dashed lines: characteristics of the uncorrected (3a) and corrected (3b) models. Grey stars: values used in the identification signals.

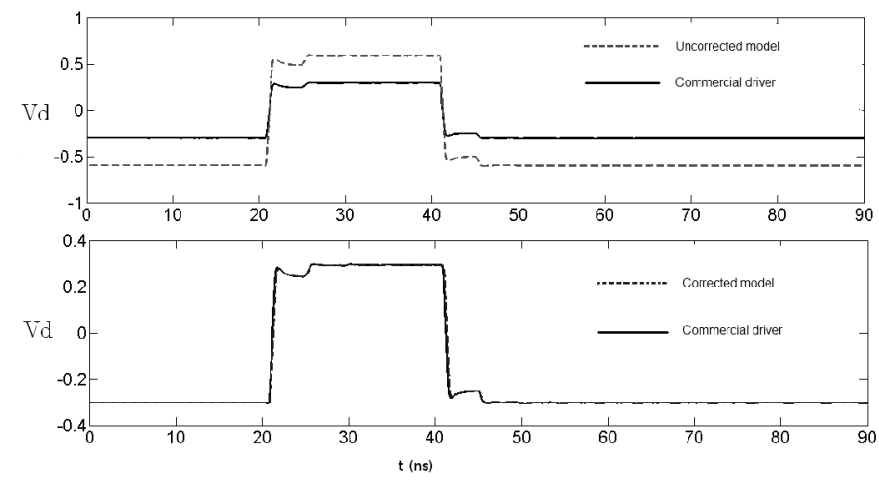

Fig. 4. Differential-voltage transient response of uncorrected (4a) and corrected (4b) models versus the original.

\section{Appendix}

The expression of a two input Volterra-Laguerre series as used in the identification of differential drivers is 


$$
\begin{aligned}
y(k)= & \sum_{i_{1}=0}^{\infty} C_{i_{1}}^{(1)} \bar{\phi}_{i_{1}}^{(1)}(k) \\
& +\sum_{i_{1}=0}^{\infty} C_{i_{1}}^{(2)} \bar{\phi}_{i_{1}}^{(2)}(k) \\
& +\sum_{i_{1}=0}^{\infty} \sum_{i_{2}=0}^{\infty} C_{i_{1}, i_{2}}^{(1,1)} \bar{\phi}_{i_{1}}^{(1)}(k) \bar{\phi}_{i_{2}}^{(1)}(k) \\
& +\sum_{i_{1}=0}^{\infty} \sum_{i_{2}=0}^{\infty} C_{i_{1}, i_{2}}^{(1,2)} \bar{\phi}_{i_{1}}^{(1)}(k) \bar{\phi}_{i_{2}}^{(2)}(k) \\
& +\sum_{i_{1}=0}^{\infty} \sum_{i_{2}=0}^{\infty} C_{i_{1}, i_{2}}^{(2,1)} \bar{\phi}_{i_{1}}^{(2)}(k) \bar{\phi}_{i_{2}}^{(1)}(k) \\
& +\sum_{i_{1}=0}^{\infty} \sum_{i_{2}=0}^{\infty} C_{i_{1}, i_{2}}^{(2,2)} \bar{\phi}_{i_{1}}^{(2)}(k) \bar{\phi}_{i_{2}}^{(2)}(k) \\
& +\ldots
\end{aligned}
$$

where $\bar{\phi}_{i}^{(1)}(k)=\phi_{i}(k) * x^{(1)}(k)$ and $\bar{\phi}_{i}^{(2)}(k)=\phi_{i}(k) * x^{(2)}(k)$.

Circuits and Systems, Vol. 32, No. 8, pp. 851-853, August 1985 .

\section{Acknowledgements}

The authors would like to acknowledge the support provided by the Institut Supérieur des Sciences et Technologie de Brest (ISSTB).

\section{References}

[1] Yi Cao, Runtao Ding, Qi-Jun Zhang, "State-Space Dynamic Neural Network Technique for High-Speed IC Applications: Modeling and Stability Analysis," IEEE Transactions on Microwave Theory and Techniques, Vol. 54, No. 6, pp. 2398-2409, June 2006.

[3] I. S. Stievano, I. A. Maio, F. G. Canavero, "M $\pi \log$ Macromodeling via Parametric Identification of Logic Gates," IEEE Transactions on Advanced Packaging, Vol. 27, No. 1, pp. 15-23, Feb. 2004.

[3] B. Mutnury, M. Swaminathan, J. P. Libous, "Macromodeling of nonlinear digital I/O drivers," IEEE Transactions on Advanced Packaging, Vol. 29, No. 1, pp. 102-113, Feb. 2006.

[4] M.G. Telescu, I.S. Stievano, F.G. Canavero, N. Tanguy, "An Application of Volterra Series to IC Buffer Models", 14th IEEE Workshop on Signal Propagation On Interconnects, Hildesheim, Germany, May 9-12, 2010

[5] R. J.G.B. Campello, G. Favier, "Optimal Expansions of Discrete-Time Volterra Models Using Laguerre Functions," Automatica, Vol. 40, No. 5, pp. 815-822, May 2004.

[6] A. Y. Kibangou, G. Favier, M. M. Hassani, "LaguerreVolterra Filters Optimization Based on Laguerre Spectra," Journal on Applied Signal Processing, Vol. 2005, No. 17, pp. 2874-2887, 2005.

[7] L.C. Calvez, P. Vilbé, P. Glouannec, "Orthonormal and nonorthonormal least squares approximation of a function subject to linear equality constraints" IEEE Trans. on 